\title{
Identification of potential causal variants for premature ovarian failure by whole exome sequencing
}

\author{
Haengun Jin, JuWon Ahn, YoungJoon Park, JeongMin Sim, Han Sung Park, Chang Soo Ryu, Nam Keun Kim \\ and KyuBum Kwack ${ }^{*}$ D
}

\begin{abstract}
Background: Premature ovarian failure (POF) is a highly heterogeneous disorder that occurs in $1 \%$ of women of reproductive age. Very few causative genes and variants contributing to POF have been detected, and the disease remains incompletely understood. In this study, we used whole exome sequencing (WES) to identify potential causal variants leading to POF.

Methods: WES was conducted to identify variants in 34 Korean patients with POF, alongside 10 normal controls. Detected variants were filtered using a range of characterized bioinformatics analyses, and the machine learning tools, CADD and VEST, were used to predict pathogenic variants that could cause disease. VarSome was used for a comprehensive interpretation of the variants. Potential causal variants finally screened by these analyses were confirmed using Sanger sequencing.

Results: We identified nine potential causative variants in genes previously associated with POF in 8 of 34 (24\%) Korean patients by WES variant analysis. These potentially pathogenic variants included mutations in the MCM8, MCM9, and HFM1 genes, which are involved in homologous recombination, DNA repair, and meiosis, and are established as causing POF. Using a combination of CADD and VEST, 72 coding variants were also identified in 72 genes, including ADAMTSL1 and FER1L6, which have plausible functional links to POF.

Conclusions: WES is a useful tool to detect genetic variants that cause POF. Accumulation and systematic management of data from a number of WES studies in specialized groups of patients with POF (family data, severe case populations) are needed to better comprehend the genetic landscape underlying POF.
\end{abstract}

Keywords: Premature ovarian failure, Whole exome sequencing, MCM8, MCM9, HFM1, Gene variants

\section{Background}

When women are around 50 years old, ovarian function generally decreases, secretion of the female hormone estrogen declines, and menopause occurs. Menopause increases the incidence of various diseases, such as hypertension, depression, and obesity [1], with

*Correspondence: kbkwack@cha.ac.kr

Department of Biomedical Science, CHA University, Gyeonggi-do 13488,

Republic of Korea consequences for wider society. Against this background, premature ovarian failure (POF) is receiving increasing attention. POF, sometimes known as premature ovarian insufficiency, refers to the loss of ovary function before 40 years-of-age. POF occurs in $1 \%$ of all women, with $0.1 \%$ experiencing POF before 30 yearsof-age [2]. POF is defined as amenorrhea, with at least 4 months loss of ovarian function, before 40 yearsof-age and levels of follicle stimulating hormone 
$(\mathrm{FSH})>40 \mathrm{IU} / \mathrm{L}$ and estradiol $<50 \mathrm{pg} / \mathrm{mL}$ (measured twice, at least a month apart, by blood test) [3, 4].

Possible causes of POF include chromosomal and genetic abnormalities, autoimmune disease, viral infectious diseases, and congenital uterine anomalies. Further, surgery due to endometriosis, ovarian cancer, and chemotherapy are also risk factors for POF. Chromosomal abnormalities are detected in $10-15 \%$ of patients with POF and other associated genetic factors are detected in $20-25 \%$ [5], while $68 \%$ of POF cases have an idiopathic character, with no apparent cause, making it difficult to identify the exact etiology.

In the last decade, variants that may be causative for POF have been discovered using various methods. Some were identified in candidate genes for POF with known roles in various relevant processes, such as genital development, DNA replication, meiosis, immune function, and metabolic function [6]. Next generation sequencing (NGS) is a powerful tool to generate accurate results by analysis of complex genetic systems. Whole exome sequencing (WES) is an NGS approach that can be applied for discovery of rare variants within protein encoding gene regions that can cause changes in protein structure [7-9]. There have been no comprehensive WES studies of patients with POF in Korea.

Recently, various methods integrating machine learning for predicting the deleteriousness of variants have been developed, based on advances in computing performance. There are three main categories of mutation risk prediction methods: (1) prediction by phylogeny and sequence structure, (2) predictions based on evolutionary conservation of sequences, and (3) machine learning-based prediction methods that integrate data from various sources [10]. In this study, two methods, Variant Effect Scoring Tool (VEST) [11] and Combined Annotation Dependent Depletion (CADD) [12], were used to predict pathogenic variants. Ranked CADD values have been widely applied to predict and classify the deleteriousness of variants in several diseases [13, 14]. VEST, which can measure the deleteriousness of sequence insertions and deletions, is also established as a tool for variant classification [15]. For interpreting deleteriousness of variants, VarSome is an impact analysis tool for human genetic variation, providing a powerful analytical resource and a repository for accumulated global knowledge of the genomics community [16].

In this study, we analyzed WES data from 34 Korean patients with POF and 10 normal controls to identify potential causal variants. Bioinformatics analysis was used to characterize protein coding sequence variants corresponding to 131 known POF-candidate genes selected from public databases. We also describe novel variants that have not previously been associated with POF.

\section{Method \\ Subjects}

Unrelated patients $(n=37)$ diagnosed with POF with amenorrhea for at least 6 months before the age of 40 years, and with FSH plasma levels $>40 \mathrm{IU} / \mathrm{L}$, were included in this study. WES was performed on 37 patients with POF (P1-P37) reported in a previous study, as well as ten normal control samples collected from postmenopausal women $\geq 48$ years old who had not had POF and had no known genetic disease. All patients with POF were of Korean ethnicity and recruited from Seoul CHA Hospital. This study was approved by the Institutional Review Boards at CHA University and the Institutional Review Boards of CHA Bundang Medical Center.

\section{Whole exome sequencing}

Genomic DNA (gDNA) was isolated from patient peripheral blood using the high-salt buffer method. A minimum of $100 \mathrm{ng}$ gDNA per sample was used to generate sequencing libraries. Genomic DNA was fragmented to 150-200 bp using an E220 Focused-ultrasonicator (Covaris, Woburn, MA, USA). After fragmentation, libraries were prepared using Agilent's SureSelect XT Human All Exon V4+UTRs Enrichment kit, according to the manufacturer's recommendations (Agilent Technologies, Santa Clara, CA, USA). Paired-end sequencing (100 bp) was performed on the HiSeq2500 platform, according to the manufacturer's instructions (Illumina Inc., San Diego, CA, USA). FastQC software (version 0.11.4) was used to assess read quality, and Trimmomatic (version 0.32 ) was used to remove low quality reads (base quality $<20)$. Sequence reads were aligned into human reference genome (hg19/GRCh37) using BWA-MEM (version 0.7.7) [17] and converted into BAM files using SAMtools (version 1.3) [18]. Sorting and indexing of BAM files was also conducted using SAMtools, and Picard (version 1.14) was used to remove duplicate reads. The mean alignedread depth value on target regions (approximately $70 \mathrm{Mb}$ ) was approximately $75 \times($ range, $50-70 \times)$ with more than $68 \%$ of target regions covered by at least $20 \times$, and $57 \%$ covered by at least $30 \times$. Single nucleotide variant (SNV) and insertion/deletion (InDel) variants were called using the GATK (version 4.0.7.0) multiple-sample analysis pipeline (HaplotypeCaller, CombineGVCFs) and functionally annotated using SnpEffect (version 4.2), as well as in-house scripts.

\section{Annotation and variants filtering}

Preprocessing, variant calling, prediction of deleterious variants, and functional annotation were conducted 
to identify potential POF causative variants (Fig. 1). Only SNVs (non-synonymous, stop gain, stop loss, and splice sites) and InDel (frameshift, non-frameshift) variants that corresponded to coding regions and may affect protein function were collated. The obtained variants were filtered by location (target regions of the SureSelect XT Human All Exon V4+UTR) and quality (read depth $\geq 20$, variant allele frequency $\geq 0.25$, genotype quality $\geq 20$ ). Due to negative selection, deleterious variants are expected to have a lower frequency than neutral mutations. This theory has been proven in past large human genome studies [19]; therefore, variants were also filtered according to minor allele frequency (MAF) $>1 \%$ in the 1000 Genomes, Genome Aggregation Database (gnomAD), and Exome Aggregation Consortium (ExAC) databases $[20,21]$ to identify deleterious changes. In addition, variants in the Korean Reference Genome Database (KRGDB) [22] with MAF $>1 \%$ were filtered to remove common variants in the ethnic Korean population. Variants found in both control and patient samples were also filtered. Gene-disease association databases were used to create a list of genes associated with POF (Additional file 1: Table S1), and variants mapping to those genes were selected as potential variants causative of POF. Identified candidate variants were finally confirmed using the Integrative Genomics Viewer. In addition, the aggregated knowledge-based tool, VarSome, was used to comprehensively review variants [23].

\section{Pathogenicity prediction of variants}

To reduce the range of candidate variants, variants were filtered using population allele frequency databases (ExAC, gnomAD, 1K genome, and KRGDB) and non-synonymous variants, predicted to result in protein modifications, were selected. To narrow the search even further and identify deleterious variants, additional annotation was conducted using the prediction tools CADD (version 1.4) and VEST (version 4). CADD is an ensemble method that integrates multiple annotations to predict the potential pathogenicity of variants, and is trained to identify potential pathogenic variants by integrating and combining several different annotation algorithms, based on a logistic regression model. VEST uses a training set, based on a random forest model, to distinguish pathogenic variants using databases of mutations classified as positive or negative. Both methods use machine learning-based algorithms and can be used to measure the deleteriousness of various types of short variants (missense SNV, nonsense SNV, splice site SNV, frameshift InDel, and non-frameshift InDel). A CADD (PHRED-like scaled) score $\geq 20$ indicates that a substitution is predicted to be among the $1 \%$ most deleterious

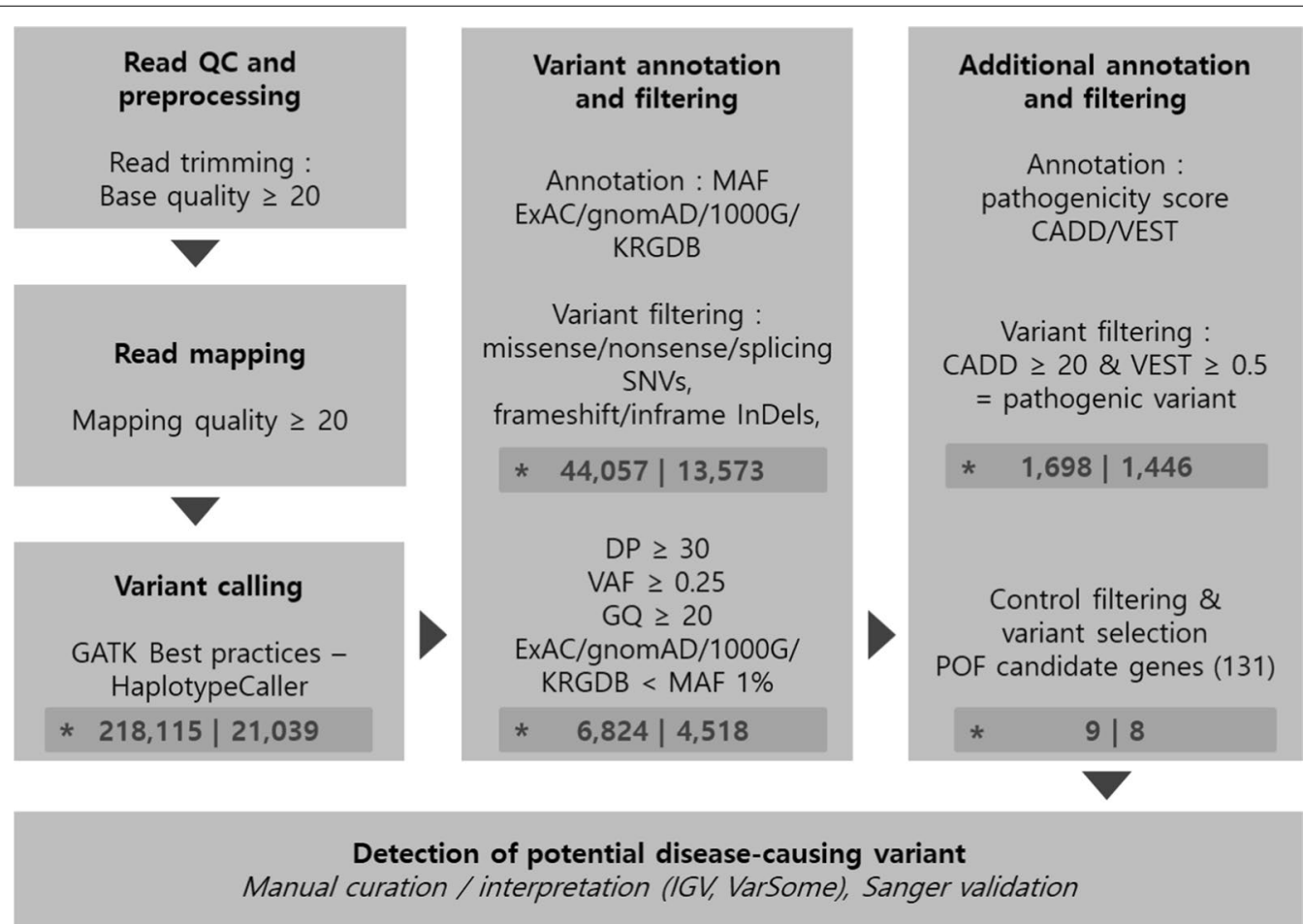

Fig. 1 WES analysis pipeline. Variant filtering was performed at each stage of WES analysis. Variant counts and gene counts are shown at each stage ${ }^{*}$ ). DP read depth, VAF variant allele frequency, GQ genotype quality 


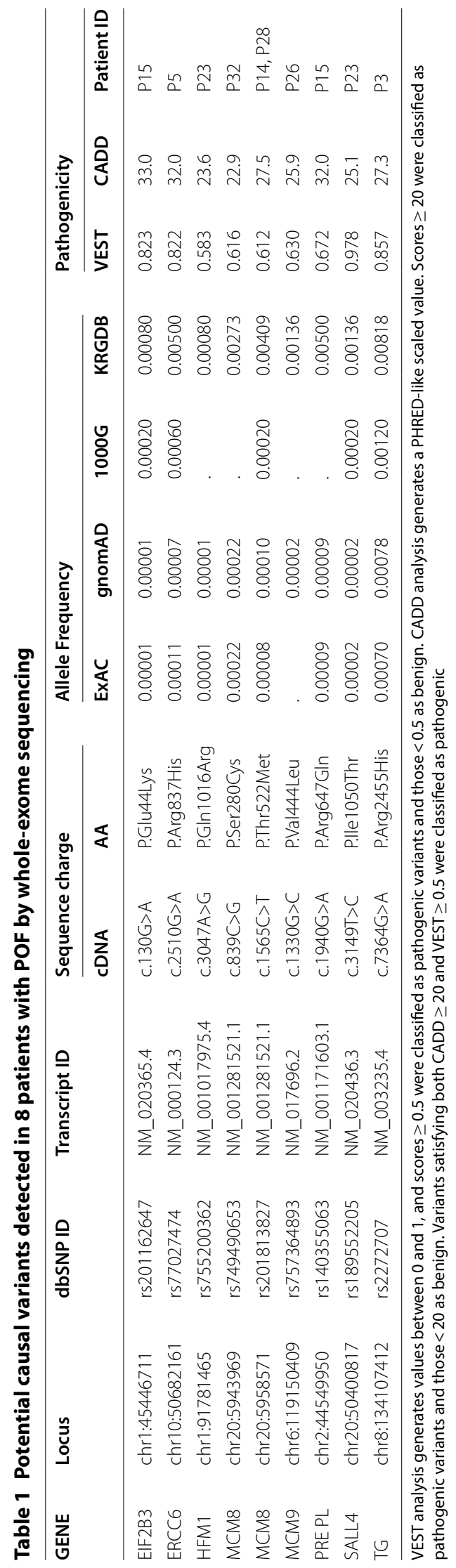


in the human genome. Therefore, CADD variants with scores $\geq 20$ were classified as pathogenic variants and those with scores $<20$ as benign. VEST scores were calculated using the CRAVAT web server (https://www. cravat.us). Variants with VEST scores $\geq 0.5$ were classified as pathogenic and those with scores $<0.5$ as benign. Finally, variants with both CADD scores $\geq 20$ and VEST scores $\geq 0.5$ were classified as potentially pathogenic candidate variants.

\section{Sanger validation}

After filtering against multiple databases, candidate variants were re-sequenced using Sanger sequencing using an Applied Biosystems 3730xl DNA Analyzer. Targeted primers were designed using Primer3 software [24] (Additional file 1: Table S2). SeqMan 7.0 (DNAStar Lasergene, USA) was used for variant analysis of Sanger sequencing data.

\section{Result}

To find potential causal variants of POF, we conducted WES using DNA from 37 patients diagnosed with POF and ten controls. Patients P1 and P31 were identified as XY females and data from patient P4 had biased GC content; therefore, data from 34 patients were included in our sequencing analysis [16]. Unfortunately, additional clinical information, such as XY karyotyping and ultrasound findings, were not available for these patients. Patient exomes were sequenced to an average sequencing depth of $100 \times$. Clinical interpretation and evidence for the variants were carefully reviewed through VarSome.

\section{Identification of variants in genes previously associated with POF}

Screening of genes known to cause POF (Additional file 1: Table S1) led to identification of nine plausible heterozygous variants (Table 1 ). These nine pathogenic variants in genes known to contribute to POF were carried by 8 of the 34 individuals (24\%). Variants in MCM8 (NM_001281521.1; c.839C>G; p.Ser280Cys, NM_001281521.1; c.1565C>T; p.Thr522Met) and MCM9 (NM_017696.2; c.1330G>C; p.Val444Leu) were identified in 4 of 34 patients. A very rare variant (ExAC; $\mathrm{MAF}=8 . \mathrm{E}-06$ ) in EIF2B3 (NM_020365.4; c.130G $>$ A; p.Glu44Lys) was detected in P15, who also carried a variant in the PREPL gene (NM_001171603.1; c.1940G>A; p.Arg647Gln). P5 had an ERCC6 variant (NM_000124.3; c.2510G>A; p.Arg837His) and an HFM1 variant (NM_001017975.4; c.3047A>G; p.Gln1016Arg), which was also a very low frequency allele (ExAC; $M A F=8$. E-06) found in P23. In addition, SALL4 (NM_020436.3; c.3149T>C; p.Ile1050Thr) and TG (P3; NM_003235.4; c.7364G>A; p.Arg2455His) variants were identified.
The genes corresponding to the nine identified variants included participants in key biological processes, such as homologous recombination, DNA repair, and meiosis (MCM8, MCM9, and HFM1). The nine potential causal variants were confirmed by Sanger sequencing (Additional file 2: Figure S1).

\section{Identification of novel, candidate variants associated with POF}

In addition to nine variants in genes associated with POF, 72 variants in 72 genes were found in two or more individuals in genes not previously associated with POF (Tables S3, S4). Of these, genes of particular interest, based on their known functions, included identical variants in two individuals found in ADAMTSL1 and FER1L6, which are genes associated with the development of reproductive organs and folliculogenesis. The ADAMTS Like 1 (ADAMTSL1) variant (NM_001040272.5; c.397G>A; p.Glu133Lys) was found in two individuals. This variant generated high risk scores using both VETS (0.924) and CADD (32.0). The variant in FER1L6 was c.814G>A; p.Gly272Ser (NM_001039112.2).

\section{Discussion}

In this study, we recruited 34 Korean patients clinically diagnosed with POF and performed WES to identify pathogenic variants. Nine variants were detected that corresponded to genes known to potentially cause POF. Among these genes, Minichromosome maintenance 8 (MCM8) and Minichromosome maintenance 9 (MCM9) are implicated in homologous recombination and repair of DNA double-strand breaks. Female mice with $M C M 8$ and MCM9 defects exhibit early oocyte loss or severe early proliferative defects in germ cells in testes [25]. The protein encoded by eukaryotic translation initiation factor 2B, subunit gamma (EIF2B3), is a subunit of the initiation factor $e I F 2 B$, which is associated with vanishing white matter (VWM) disease and leukodystrophy. Ovarian failure is frequently reported in women with VWM disease [26]. Mutations of the EIF2B3 gene are very rarely found in patients with POF [27]. Mutations in prolyl endopeptidase like (PREPL) are associated with hypotonia-cystinuria syndrome (HCS), which is caused by deletions including SLC3A1. PREPL deficiency causes hypergonadotropic hypogonadism and HCS [28]. The ERCC6-PGBD3 fusion protein and mutations in the ERCC excision repair 6 (ERCC6) gene are reported to cause POF and are among several proteins that participate in, or regulate, DNA repair [29]. The protein encoded by helicase for meiosis 1 (HFM1) is thought to be an ATP-dependent DNA helicase and is primarily expressed in germ-line cells, such as testis and ovary. Mutations in the HFM1 gene, which encodes a protein 
necessary for homologous recombination and synapsis during meiosis, are a cause of POF [30]. Spalt like transcription factor 4 (SALL4) plays important roles in maintaining the pluripotency of embryonic stem cells and regulating the embryonic development of many organisms. Similar to adult mice, SALL4 is highly expressed in adult humans, with expression restricted to the testis and ovary [31]. A recent study of 50 patients with POF reported pathogenic mutations in SALL4 (3/50 patients, p.Val181Met; p.Lys597Arg; p.Thr760Ile) and predicted that these variants had different effects on SALL4 activity [32]. The SALL4 variant (NM_020436.3; c.3149T>C; p.Ile1050Thr) detected in our study was not one of the three previously reported mutations; however, it could be expected to also influence $S A L L 4$ activity. Thyroglobulin (TG) is a precursor of the iodinated thyroid hormones, thyroxine (T4) and triiodothyronine (T3), and is synthesized predominantly by the thyroid gland. Mutations in $T G$ are associated with the development of thyroid disorders, such as congenital hypothyroidism, thyroid cancer, and autoimmunity [33]. Previously, we reported that hypothyroidism due to TG mutations could lead to POF. [34, 35]. The TG variant (NM_003235.4; c.7364G>A; p.Arg2455His) identified in P3 may have resulted in hypothyroidism.

We also reported identical variants that did not correspond to known POF genes but were found in two or more individuals with POF (Additional file 1: Table S4). The ADAMTS protease family contributes to aspects of reproductive organ development and tissue morphogenesis, and dysregulation or functional alteration of ADAMTS proteins are associated with reproductive disorders, such as polycystic ovary syndrome and POF [36]. In chickens, expression of ADAMTSL1 increases in the developing ovaries during gonadal differentiation [37]. FER1L6 homologs in model organisms are involved in folliculogenesis and a copy number variant was reported in a patient with POF [38]. Solute carrier family $3 \mathrm{mem}-$ ber 1 (SLC3A1) frameshift variant (NM_000341.3; c.1820delT; p.Leu607fs) has very low allele frequency in both the ExAC $(\mathrm{MAF}=8.24 \mathrm{E}-06)$ and gnomAD (4.08E-06) databases. According to VarSome, this SLC3A1 variant has a strong association with Cystineuria. Cystineuria is a rare hereditary disease associated with kidney stones, which is primarily caused by mutations in two protein subunits (rBAT and $\mathrm{b}^{0,+} \mathrm{AT}$ ) encoded by SLC3A1 and SLC7A9 [39].

POF is a highly heterogeneous disorder that can be caused by a variety of factors, suggesting that an unspecified number of variants can cause the condition. The increase of research use of NGS has led to the discovery of numerous disease-specific genes and variants in patients with POF; however, these results are rarely verified through sufficient downstream experiments and are not managed systematically, making in-depth study of the disease difficult. To establish the genetic pathogenesis of POF, efforts are needed to understand the complex genetic mechanisms involved; for example, using integrated databases and approaches to predict combinations of disease-associated variants.

\section{Conclusion}

Sporadic POF is a highly heterogeneous condition with no common genetic cause. Hence, the scope for identifying genes and variants common to many individuals is limited. Here, we identified several variants and genes that may cause disease in 34 Korean patients with apparent isolated POF. We report novel candidate variants in ADAMTSL1 and FER1L6, as well as alterations in genes previously reported to be associated with POF. In the study of disease, prediction of the risk associated with variants detected in patients by WES and in the candidate genes associated with the disease is a useful approach to find causative genetic variants. Our data confirm that WES is an efficient tool for studying the genetic causes of POF and can contribute to understanding of disease etiology. For more accurate identification of pathogenic variants, integration and systematic management of data collected from a number of WES studies of specific POF populations (e.g., family data and severe case populations) will be necessary.

\section{Supplementary information}

Supplementary information accompanies this paper at https://doi. org/10.1186/s12920-020-00813-x.

\begin{abstract}
Additional file 1: Table S1. List of POF/POI genes (131). List of candidate POF genes obtained from four public databases (DisGeNET, Monarch, MalaCards and NCBI:Gene) that collect genes and variants associated with human disease. Table S2. Primers used for confirming selected variants. Table S3. Genes with identical rare variants (MAF $\leq 0.01)$ detected in more than one individual. Table S4. Identical coding variants found in two or more individuals via whole-exome sequencing. VEST analysis generates values between 0 and 1 , and scores $\geq 0.5$ were classified as pathogenic variants and those $<0.5$ as benign. CADD analysis generates a PHREDlike scaled value. Scores $\geq 20$ were classified as pathogenic variants and those $<20$ as benign. Variants satisfying both CADD $\geq 20$ and VEST $\geq 0.5$ were classified as pathogenic.

Additional file 2: Figure S1. Sanger validation. Sanger sequencing chromatogram showing nine candidate variants and one novel variant in ten patients with POF. Variant positions are indicated by $\left(^{*}\right)$. Images were extracted using SeqScanner v2 (Applied Biosystems, Foster City, CA, USA). Peaks, bases, and quality bars are shown. See SeqScanner v2 help for details.
\end{abstract}

\section{Abbreviations}

POF: Premature ovarian failure; NGS: Next generation sequencing; WES: Whole exome sequencing; SNV: Single nucleotide variant; InDel: Insertion/deletion variant; MAF: Minor allele frequency; FSH: Follicle stimulating hormone; gDNA: Genomic DNA; VWM: Vanishing white matter; HCS: Hypotonia-cystinuria syndrome. 


\section{Acknowledgements}

We thank all those who have given us advice and help with our manuscript.

\section{Authors' contributions}

In this study, HJ performed WES analysis, contributed to scientific discussion, and wrote the paper. JS helped with the Sanger validation experiments. JA and YP participated in the variant calling analysis. HP, CR and NK performed the collection, quality control (QC) and sequence alignment analysis of controls. Professor KK designed all of the WES analysis, as well as participating in scientific discussion. All authors read and approved the final manuscript.

\section{Funding}

This study was supported by the Korea Ministry of Environment (MOE) as the Environmental Health Action Program (201600136008) and a grant of the Korea Healthcare Technology R\&D Project, Ministry of Health and Welfare, Republic of Korea (H18C1999). The funding bodies had no role in study design, collection, analysis, and interpretation of data and in writing the manuscript.

\section{Availability of data and materials}

All data generated or analyzed in this study is included in published articles. Human genome version hg19/GRCh37 was used as a reference genome in this study and is available in the USCS Genome Browser website (https:// hgdownload.soe.ucsc.edu/goldenPath/hg19/bigZips/hg19.fa.gz). VarSome (https://varsome.com/) was used for a comprehensive interpretation of variants. The sequence data of patients are available in the NCBI SRA under the accession number PRJNA668627. The sequence data of controls are available in the NCBI SRA under the accession number PRJNA601005. The controls used in this study are 10 control samples from PRJNA601005, and the NCBI SRA accession numbers are SAMN13840382, SAMN13840383, SAMN13840393, SAMN13840395, SAMN13840396, SAMN13840398, SAMN13840400, SAMN13840387, SAMN13840386 and SAMN13840385.

\section{Ethics approval and consent to participate}

This study was approved by the Institutional Review Boards (IRB) of CHA University (1044308-201310-BR-002-01) and the Institutional Review Boards of CHA Bundang Medical Center (ID No. 2010-01-123). All patients provided a written informed consent for their participation in the study and written consent was obtained from all participants. Among samples collected prior to 20050101, anonymous participants who were unable to obtain additional written notice consent and publication consent were exempted from obtaining written notice consent and publication consent in accordance with the Bioethics Law regulations in effect in 20050101. (https://doi.org/10.3343/ Imo.2015.5.3.113).

\section{Consent for publication}

Not applicable.

\section{Competing interests}

The authors declare that they have no competing interests.

Received: 5 January 2020 Accepted: 21 October 2020

Published online: 27 October 2020

\section{References}

1. Ford ES, Li C, Zhao G, Tsai J. Trends in obesity and abdominal obesity among adults in the United States from 1999-2008. Int J Obes (Lond). 2011;35(5):736-43.

2. Shelling AN. Premature ovarian failure. Reproduction. 2010;140(5):633-41.

3. Welt CK. Primary ovarian insufficiency: a more accurate term for premature ovarian failure. Clin Endocrinol (Oxf). 2008;68(4):499-509.

4. Norling A, Hirschberg AL, Rodriguez-Wallberg KA, Iwarsson E, Wedell A, Barbaro M. Identification of a duplication within the GDF9 gene and novel candidate genes for primary ovarian insufficiency (POI) by a customized high-resolution array comparative genomic hybridization platform. Hum Reprod. 2014;29(8):1818-27.
5. Qin Y, Jiao X, Simpson JL, Chen ZJ. Genetics of primary ovarian insufficiency: new developments and opportunities. Hum Reprod Update. 2015;21(6):787-808.

6. Tucker EJ, Grover SR, Bachelot A, Touraine P, Sinclair AH. Premature ovarian insufficiency: new perspectives on genetic cause and phenotypic spectrum. Endocr Rev. 2016;37(6):609-35.

7. Caburet S, Arboleda VA, Llano E, Overbeek PA, Barbero JL, Oka K, Harrison W, Vaiman D, Ben-Neriah Z, Garcia-Tunon I, et al. Mutant cohesin in premature ovarian failure. N Engl J Med. 2014;370(10):943-9.

8. de Vries L, Behar DM, Smirin-Yosef P, Lagovsky I, Tzur S, Basel-Vanagaite L. Exome sequencing reveals SYCE1 mutation associated with autosomal recessive primary ovarian insufficiency. J Clin Endocrinol Metab. 2014;99(10):E2129-2132.

9. AlAsiri S, Basit S, Wood-Trageser MA, Yatsenko SA, Jeffries EP, Surti U, Ketterer DM, Afzal S, Ramzan K, Faiyaz-UI Haque M, et al. Exome sequencing reveals MCM8 mutation underlies ovarian failure and chromosomal instability. J Clin Invest. 2015;125(1):258-62.

10. Li J, Zhao T, Zhang Y, Zhang K, Shi L, Chen Y, Wang X, Sun Z. Performance evaluation of pathogenicity-computation methods for missense variants. Nucleic Acids Res. 2018;46(15):7793-804.

11. Carter H, Douville C, Stenson PD, Cooper DN, Karchin R. Identifying Mendelian disease genes with the variant effect scoring tool. BMC Genomics. 2013;14(Suppl 3):S3.

12. Kircher M, Witten DM, Jain P, O'Roak BJ, Cooper GM, Shendure J. A general framework for estimating the relative pathogenicity of human genetic variants. Nat Genet. 2014;46(3):310-5.

13. Nakagomi H, Mochizuki H, Inoue M, Hirotsu Y, Amemiya $K$, Sakamoto I, Nakagomi S, Kubota T, Omata M. Combined annotation-dependent depletion score for BRCA1/2 variants in patients with breast and/or ovarian cancer. Cancer Sci. 2018;109(2):453-61.

14. Nomura A, Tada H, Teramoto R, Konno T, Hodatsu A, Won HH, Kathiresan $\mathrm{S}$, Ino H, Fujino N, Yamagishi M, et al. Whole exome sequencing combined with integrated variant annotation prediction identifies a causative myosin essential light chain variant in hypertrophic cardiomyopathy. J Cardiol. 2016;67(2):133-9.

15. Lanktree MB, Haghighi A, Guiard E, Iliuta IA, Song X, Harris PC, Paterson $A D$, Pei Y. Prevalence estimates of polycystic kidney and liver disease by population sequencing. J Am Soc Nephrol. 2018;29(10):2593-600.

16. Kopanos C, Tsiolkas V, Kouris A, Chapple CE, Albarca Aguilera M, Meyer $\mathrm{R}$, Massouras A. VarSome: the human genomic variant search engine. Bioinformatics. 2019;35(11):1978-80.

17. Lee Y, Kim C, Park Y, Pyun JA, Kwack K. Next generation sequencing identifies abnormal $Y$ chromosome and candidate causal variants in premature ovarian failure patients. Genomics. 2016;108(5-6):209-15.

18. Li H, Durbin R. Fast and accurate long-read alignment with BurrowsWheeler transform. Bioinformatics. 2010;26(5):589-95.

19. Li H, Handsaker B, Wysoker A, Fennell T, Ruan J, Homer N, Marth G, Abecasis $G$, Durbin R. Genome project data processing $S$ : the sequence alignment/map format and SAMtools. Bioinformatics. 2009;25(16):2078-9.

20. Tennessen JA, Bigham AW, O'Connor TD, Fu W, Kenny EE, Gravel S, McGee S, Do R, Liu X, Jun G, et al. Evolution and functional impact of rare coding variation from deep sequencing of human exomes. Science. 2012:337(6090):64-9.

21. Lek M, Karczewski KJ, Minikel EV, Samocha KE, Banks E, Fennell T, O'Donnell-Luria AH, Ware JS, Hill AJ, Cummings BB, et al. Analysis of protein-coding genetic variation in 60,706 humans. Nature. 2016:536(7616):285-91.

22. Sills ES, Brady AC, Omar AB, Walsh DJ, Salma U, Walsh AP. IVF for premature ovarian failure: first reported births using oocytes donated from a twin sister. Reprod Biol Endocrinol. 2010;8:31.

23. Jung KS, Hong KW, Jo HY, Choi J, Ban HJ, Cho SB, Chung M. KRGDB: the large-scale variant database of 1722 Koreans based on whole genome sequencing. Database (Oxford). 2020. https://doi.org/10.1093/database/ baaa030.

24. Untergasser A, Cutcutache I, Koressaar T, Ye J, Faircloth BC, Remm M, Rozen SG. Primer3-new capabilities and interfaces. Nucleic Acids Res. 2012;40(15):e115

25. Lutzmann M, Grey C, Traver S, Ganier O, Maya-Mendoza A, Ranisavljevic N, Bernex F, Nishiyama A, Montel N, Gavois E, et al. MCM8- and MCM9deficient mice reveal gametogenesis defects and genome instability due to impaired homologous recombination. Mol Cell. 2012;47(4):523-34. 
26. Fogli A, Rodriguez D, Eymard-Pierre E, Bouhour F, Labauge P, Meaney BF, Zeesman S, Kaneski CR, Schiffmann R, Boespflug-Tanguy O. Ovarian failure related to eukaryotic initiation factor $2 \mathrm{~B}$ mutations. Am J Hum Genet. 2003;72(6):1544-50.

27. Fogli A, Gauthier-Barichard F, Schiffmann R, Vanderhoof VH, Bakalov VK, Nelson LM, Boespflug-Tanguy O. Screening for known mutations in EIF2B genes in a large panel of patients with premature ovarian failure. BMC Womens Health. 2004;4(1):8.

28. Regal L, Martensson E, Maystadt I, Voermans N, Lederer D, Burlina A, Juan Fita MJ, Hoogeboom AJM, Olsson Engman M, Hollemans T, et al. PREPL deficiency: delineation of the phenotype and development of a functional blood assay. Genet Med. 2018;20(1):109-18.

29. Qin Y, Guo T, Li G, Tang TS, Zhao S, Jiao X, Gong J, Gao F, Guo C, Simpson $J$, et al. CSB-PGBD3 mutations cause premature ovarian failure. PLoS Genet. 2015;11(7):e1005419.

30. Wang J, Zhang $\mathrm{W}$, Jiang $\mathrm{H}$, Wu BL. Primary ovarian insufficiency C: mutations in HFM1 in recessive primary ovarian insufficiency. N Engl J Med. 2014;370(10):972-4.

31. Kohlhase J, Heinrich M, Schubert L, Liebers M, Kispert A, Laccone F, Turnpenny P, Winter RM, Reardon W. Okihiro syndrome is caused by SALL4 mutations. Hum Mol Genet. 2002;11(23):2979-87.

32. Wang Q, Li D, Cai B, Chen Q, Li C, Wu Y, Jin L, Wang X, Zhang X, Zhang F. Whole-exome sequencing reveals SALL4 variants in premature ovarian insufficiency: an update on genotype-phenotype correlations. Hum Genet. 2019;138(1):83-92.
33. Rubio IG, Medeiros-Neto G. Mutations of the thyroglobulin gene and its relevance to thyroid disorders. Curr Opin Endocrinol Diabetes Obes. 2009;16(5):373-8.

34. Pyun JA, Kang H, Kim J, Cha DH, Kwack K. Thyroglobulin gene is associated with premature ovarian failure. Fertil Steril. 2011;95(1):397-400.

35. Pyun JA, Kim S, Cha DH, Ko JJ, Kwack K. Epistasis between the HSD17B4 and TG polymorphisms is associated with premature ovarian failure. Fertil Steril. 2012;97(4):968-73.

36. Russell DL, Brown HM, Dunning KR. ADAMTS proteases in fertility. Matrix Biol. 2015;44-46:54-63.

37. Carre GA, Couty I, Hennequet-Antier C, Govoroun MS. Gene expression profiling reveals new potential players of gonad differentiation in the chicken embryo. PLOS ONE. 2011;6(9):e23959.

38. Ledig S, Ropke A, Wieacker P. Copy number variants in premature ovarian failure and ovarian dysgenesis. Sex Dev. 2010;4(4-5):225-32.

39. Rhodes HL, Yarram-Smith L, Rice SJ, Tabaksert A, Edwards N, Hartley A Woodward MN, Smithson SL, Tomson C, Welsh Gl, et al. Clinical and genetic analysis of patients with cystinuria in the United Kingdom. Clin J Am Soc Nephrol. 2015;10(7):1235-45.

\section{Publisher's Note}

Springer Nature remains neutral with regard to jurisdictional claims in published maps and institutional affiliations.
Ready to submit your research? Choose BMC and benefit from:

- fast, convenient online submission

- thorough peer review by experienced researchers in your field

- rapid publication on acceptance

- support for research data, including large and complex data types

- gold Open Access which fosters wider collaboration and increased citations

- maximum visibility for your research: over 100M website views per year

At BMC, research is always in progress.

Learn more biomedcentral.com/submissions 